\title{
STABILITY OF LOBED INFLATABLE STRUCTURES
}

\author{
B.A. Lennon* and S. Pellegrino ${ }^{\dagger}$ \\ Department of Engineering, University of Cambridge, \\ Trumpington Street, Cambridge, CB2 1PZ, U.K.
}

\begin{abstract}
$\underline{\text { Abstract }}$
This paper is concerned with the stability of inflatable structures that are constructed by joining together a number of gores in such a way that the membrane forms a series of bulges or lobes. Under certain conditions the nominal configuration of these structures is unstable, and hence a deployment scenario may be envisaged where the structure will never assume its intended shape. Instead, it may end up in a distorted, but more stable configuration. This paper considers two structures, a cylinder with circumferential lobes and a more general lobed spheroidal configuration, typical of high-pressure balloons. The stability of both structures is shown to depend on their geometry.
\end{abstract}

\section{Background and Introduction}

Membrane structures have a long history as sails, balloons, parachutes and decelerators. A good example of an inflatable membrane structure is the scientific balloon shown in Figure 1. Other examples are found in the parachutes and inflatable gasbags used by the Mars Pathfinder Mission ${ }^{1}$, shown in Figure 2.

This paper is concerned with inflatable membrane structures that are made from a number of segments such that the expected inflated configuration is a regular shape consisting of a series of lobes. The membrane material is assumed to be very thin, hence only tensile stresses are admissible.

Most structural designers are familiar with the criteria for the design of conventional rigid structures. Inflatable membrane structures behave differently; their flexibility makes them unable to carry compression or moments, and leads to the possibility of them having different useful configurations. It also leads to somewhat unusual problems in relation to their stability.

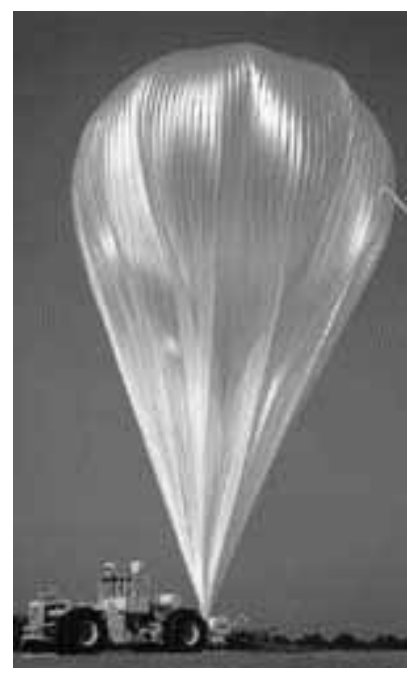

Figure 1: Scientific balloon (Courtesy of NASA).

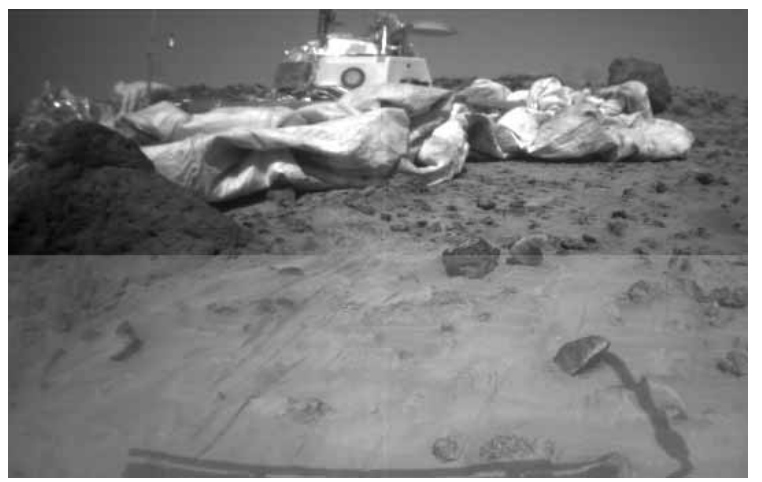

Figure 2: Mars Pathfinder Lander (Courtesy of NASA).

\footnotetext{
${ }^{*}$ Research Student.

${ }^{\dagger}$ Reader in Structural Engineering, Associate Fellow AIAA.
} Copyright (C) 2000 by B.A. Lennon and S. Pellegrino. Published by the American Institute of Aeronautics and Astronautics, Inc. with permission. 


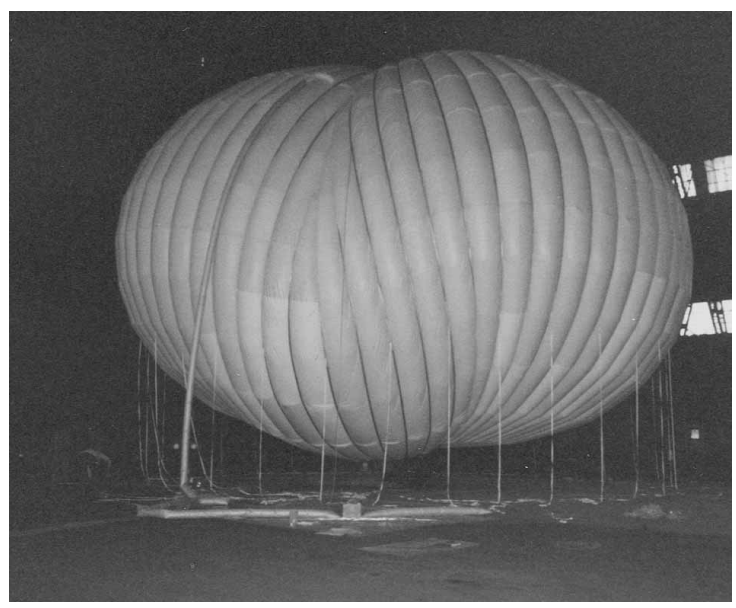

Figure 3: Endeavour balloon (Courtesy of J. Nott).

Our work on lobed inflatable structures originated from a problem with the stability of Endeavour, a high pressure balloon designed for circumnavigation of the globe by Julian Nott. The balloon originally consisted of sixty-four lobes but when inflated it adopted a non-symmetrical shape, Figure 3. Removal of two lobes and re-inflation diminished but did not eliminate the asymmetry problem. Two more lobes were removed to give a balloon with sixty lobes and on re-inflation this balloon adopted the nominal shape. This problem was studied by Calladine $^{2}$ who proposed an equivalent stiffness approach by studying the buckling of an equivalent Euler strut.

Like all structures, the stability of an inflated membrane structure depends on the potential energy of the system. This gives the following expression:

$$
E=E_{G}+E_{M}
$$

Here $E_{G}$ is the potential energy due to the gas in the balloon, hence $E_{G}=-p V$ where $p$ is the pressure (constant) and $V$ the enclosed volume. $E_{M}$ is the elastic strain energy in the membrane which will be neglected in our analysis. Hence, assuming $E \simeq-p V$, the stability of a lobed inflatable structure may be determined by examining the variation of its volume $V$ with the configuration of the structure.

This paper studies two systems to demonstrate stability problems with lobed inflatable structures. The first study is a column with circumferential lobes. This introduces some of the problems encountered in a simple three-dimensional system and the methods required to approach these problems. It also acts as an introduction to the second study, a lobed spheroid of more significant mathematical complexity.

\section{Stability of a Lobed Column}

The lobed column shown in Figure 4(a) is a simple, rather abstract example that can display unstable behaviour. A mode of buckling is shown in Figure 4(b). The column consists of $n$ identical hollow doughnut shaped elements with a central hole, joined together on the edges. The ends are sealed by two rigid plates that are held at a fixed distance by an inextensible cable $P_{1} P_{2}$ of length $n h$. A typical column is shown in Figure 4.

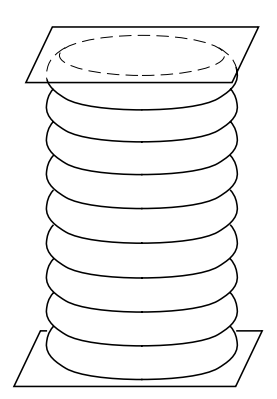

(a)

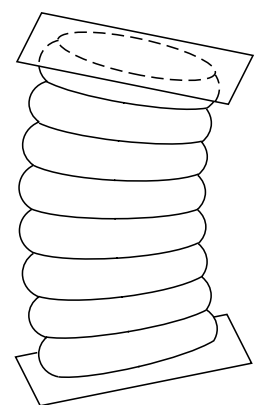

(b)
Figure 4: Lobed column in (a) nominal and (b) buckled configurations.

Vertical sections through the structure are shown in Figure 5, showing the nominal configuration, and Figure 6, showing the deformed configuration. It is assumed that the circumferential "hoops" that separate the lobed segments maintain their shape in this buckling mode. Note that the length of $P_{1} P_{2}$ remains unchanged. Also note that the centreline of the column, which no longer coincides with $P_{1} P_{2}$, is now curved and has become longer.

It will be assumed that in the buckled configuration the centreline becomes an arc of a circle. A more general shape (for example a sine wave) would be more accurate but it would also lead to unnecessary complication of the calculations.

The segments of the column distort identically if it is assumed that the axis of the cylinder deflects into an arc of a circle. Let the angle subtended by the two end plates be $2 \Psi$, as shown in Figure 6 . Let the angle subtended by the circumferential hoops at the top and bottom of each segment be $\psi$.

In the nominal configuration the structure is axisymmetric and it is easiest to determine the enclosed volume by multiplying by $2 \pi$ the area of the crosssection in Figure 5. The deformed structure is not axisymmetric, but its volume can still be derived from the area of the cross-section in Figure 6. An 
expression is derived for the cross-sectional area of a segment in the plane of deflection and this expression will be later modified to find the expression for a general segment cross-section. The segment volume will then be found by integration.

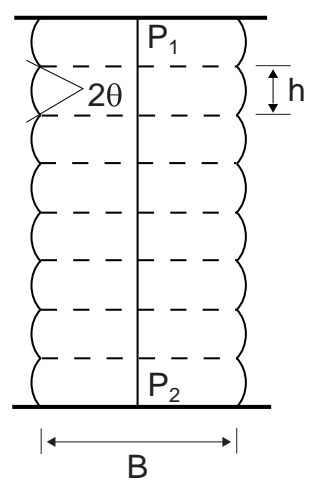

Figure 5: Cross-section of column.

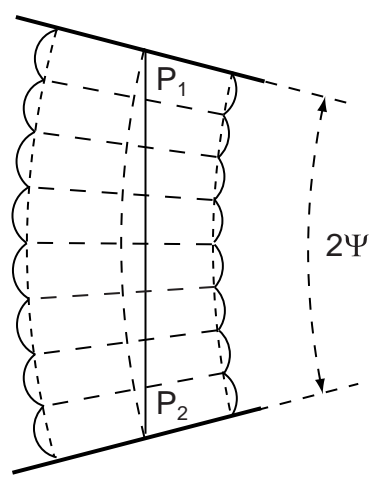

Figure 6: Cross-section of buckled column.

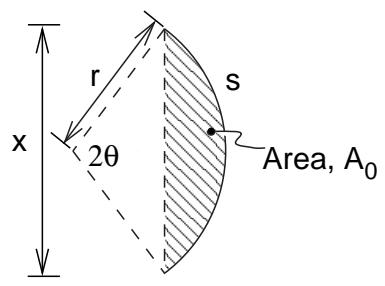

Figure 7: Vertical cross-section of a lobe.

First consider the cross-sectional area of a lobe of radius $r$ and subtending an angle $2 \theta$; the arc and chord lengths are respectively $s$ and $x$, see Figure 7 . Clearly

$$
x=\frac{s \sin \theta}{\theta}
$$

It can be shown that the area of the segment between the chord and the arc has the expression

$$
A_{0}=\frac{s^{2}}{4}\left(\frac{1}{\theta}-\frac{\cos \theta \sin \theta}{\theta^{2}}\right)
$$

Next, following Calladine ${ }^{2}$, consider the crosssection of a typical segment as shown in Figure 8 (recall that all segments are identical).

(a)

(b)

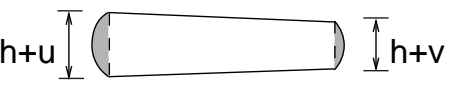

(c)

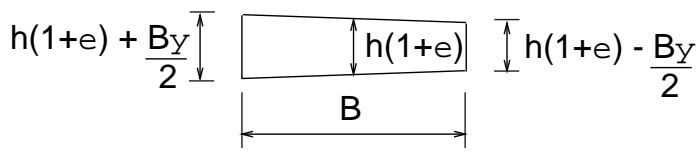

Figure 8: Cross-sections of a segment in the plane of deformation.

Figure 8(a) shows the segment in the undeformed configuration and Figure $8(\mathrm{~b})$ shows the deformed segment. Figure 8(c) shows the same deformed segment but without the lobes.

Let $\varepsilon$ be the small axial strain of the column $P_{1} P_{2}$. From Figure 6

$$
\varepsilon=\frac{n h \Psi / \sin \Psi-n h}{n h}=\frac{\Psi}{\sin \Psi}-1
$$

Noting that

$$
\Psi=\frac{L}{2 h} \psi
$$

we obtain

$$
\varepsilon=\frac{L \psi}{2 h} \frac{1}{\sin (L \psi / 2 h)}-1
$$

In Figure 8(b) the cross-sectional area of the lobes that have become elongated is denoted by $A_{1}$ while the cross-sectional area of the lobes on the shortened side is $A_{2}$. A truncated Taylor series expansion may be used to approximate $A_{1}$ and $A_{2}$ in terms of the undeformed area $A_{0}$. Then

$$
\begin{aligned}
& A_{1}=A_{0}+u A_{0}{ }^{\prime}+u^{2} A_{0}{ }^{\prime \prime} / 2+\ldots \\
& A_{2}=A_{0}+v A_{0}{ }^{\prime}+v^{2} A_{0}{ }^{\prime \prime} / 2+\ldots
\end{aligned}
$$

where $A_{0}{ }^{\prime}$ and $A_{0}{ }^{\prime \prime}$ are respectively the first and second derivatives of $A_{0}$ with respect to $x$. The following expressions were obtained by Calladine ${ }^{2}$

$$
\begin{gathered}
A_{0}^{\prime}=-\frac{s}{2} \frac{\cos \theta}{\theta} \\
A_{0}^{\prime \prime}=-\frac{1}{2} \frac{\cos \theta+\theta \sin \theta}{\sin \theta-\theta \cos \theta}
\end{gathered}
$$


Comparing Figure 8(b) to (a)

$$
h+u=h(1+\varepsilon)+B \psi / 2
$$

Substituting Equation 6 and rearranging

$$
u=\frac{L \psi}{2} \frac{1}{\sin (L \psi / 2 h)}-h+\frac{B \psi}{2}
$$

and similarly

$$
v=\frac{L \psi}{2} \frac{1}{\sin (L \psi / 2 h)}-h-\frac{B \psi}{2}
$$

Using the approximation

$$
\frac{\sin \Psi}{\Psi} \approx 1-\frac{\Psi^{2}}{6}
$$

we obtain

$$
u=\frac{h \psi^{2}}{24} \frac{L^{2}}{h^{2}}+\frac{B \psi}{2}
$$

and

$$
v=\frac{h \psi^{2}}{24} \frac{L^{2}}{h^{2}}-\frac{B \psi}{2}
$$

The change in cross-sectional area of a lobe segment $\Delta A_{s}$ may be evaluated by taking the difference between the area of a trapezium plus $A_{1}+A_{2}$ and the area of a rectangle plus $2 A_{0}$. Hence, after simplification

$$
\begin{aligned}
\Delta A_{s}= & B(u / 2+v / 2)+A_{0}{ }^{\prime}(u+v) \\
& +A_{0}{ }^{\prime \prime}\left(u^{2}+v^{2}\right) / 2
\end{aligned}
$$

The difference in potential energy between the buckled and nominal configuration of the system, and hence its stability, depends on the change in volume $\Delta V_{s}$. Hence, the expression for $\Delta A_{s}$ is integrated over the segment to obtain $\Delta V_{s}$. Referring to Figure 9 the values of $u$ and $v$ are replaced by $u \sin \varphi$ and $v \sin \varphi$ respectively to obtain the area of a general cross-section, which is rotated through $\pi$ around the axis

$$
\begin{aligned}
\Delta V_{s}=\int_{0}^{\pi}\left[B \frac{u+v}{2} \sin \varphi+A_{0}{ }^{\prime}(u+v) \sin \varphi\right. \\
\left.+A_{0}{ }^{\prime \prime} \frac{u^{2}+v^{2}}{2} \sin ^{2} \varphi\right] d \varphi \\
\frac{\mathrm{u}}{2} \sin \varphi
\end{aligned}
$$

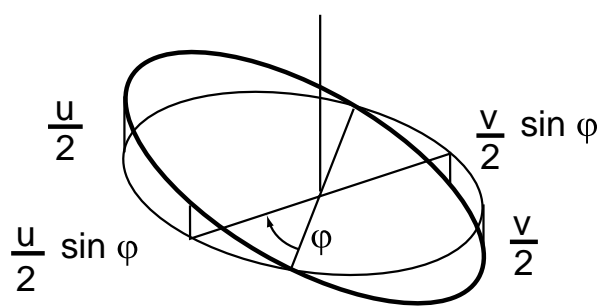

Figure 9: Definition of deformation parameters.
The change in volume must be negative for the system to be stable, implying that Equation 18 must be negative. Equations 15 and 16 may be used to evaluate $u+v$ and $u^{2}+v^{2}$

$$
u+v=2\left(\frac{h \psi^{2}}{24}\right)\left(\frac{L}{h}\right)^{2}
$$

$$
u^{2}+v^{2}=\left(\frac{h \psi^{2}}{24}\right)^{2}\left(\frac{L}{h}\right)^{4}+\frac{B^{2} \psi^{2}}{4}
$$

Substituting back into Equation 18, ignoring higher order terms, and setting $\Delta V_{s}<0$

$$
\begin{array}{r}
\int_{0}^{\pi}\left[B \frac{h \psi^{2}}{24}\left(\frac{L}{h}\right)^{2} \sin \varphi+2 A_{0}^{\prime} \frac{h \psi^{2}}{24}\left(\frac{L}{h}\right)^{2} \sin \varphi\right. \\
\left.+A_{0}^{\prime \prime} \frac{B^{2} \psi^{2}}{4} \sin ^{2} \varphi\right] d \varphi<0
\end{array}
$$

Rearranging

$$
\begin{array}{r}
\frac{B \psi^{2} L^{2}}{24 h} \int_{0}^{\pi}\left(\sin \varphi+\frac{2}{B} A_{0}^{\prime} \sin \varphi\right. \\
\left.+\frac{6 h B}{L^{2}} A_{0}^{\prime \prime} \sin ^{2} \varphi\right) d \varphi<0
\end{array}
$$

Neglecting the positive factor in Equation 22 and integrating

$$
1+\frac{2}{B} A_{0}^{\prime}+\frac{3 \pi h B}{2 L^{2}} A_{0}^{\prime \prime}<0
$$

Substituting Equations 9 and 10 into Equation 23 and rearranging

$$
1-\frac{h}{B} \frac{\cos \theta}{\sin \theta}-\frac{3 \pi h B}{4 L^{2}} \frac{\cos \theta+\theta \sin \theta}{\sin \theta-\theta \cos \theta}<0
$$

It can be shown that the third term in this equation is much bigger than the second. Hence the following stability condition is obtained

$$
\frac{3 \pi}{4} \frac{h B}{L^{2}}>\frac{\sin \theta-\theta \cos \theta}{\cos \theta+\theta \sin \theta}
$$

This equation shows that stability increases with the width of the column and the height of the segments, but decreases quadratically with the length of the structure. The right hand side of Equation 25 increases with increasing $\theta$ hence less prominent lobes lead to more stable structures. This equation may also be rewritten as

$$
\frac{3 \pi}{4} \frac{a}{n}>\frac{\sin \theta-\theta \cos \theta}{\cos \theta+\theta \sin \theta}
$$

4 
where $n$ is the number of segments and $a$ is the aspect ratio $(=B / L)$ of the entire structure. Using this equation it is easy to construct columns that are unstable; for example a $1 \mathrm{~m}$ long column of width $0.3 \mathrm{~m}$ in which each has an angle of embrace $2 \theta$ of $60^{\circ}$ will buckle if twenty segments are used. A column of the same dimensions but using only fifteen segments will be stable. Note that this result is independent of the pressure in the column.

\section{Stability of a Lobed Spheroid}

This section examines the stability of a spheroidal structure with lobes as shown in Figure 10. The structure consists of sixteen gores attached to inextensible tapes along the edges. The profile of the tapes when a uniform pressure difference is applied to the structure was first studied by Taylor ${ }^{3}$ during an analysis of the shapes of parachutes. It was later extended to other kinds of decelerators ${ }^{4}$, and the same approach has been recently proposed for high pressure scientific balloons. ${ }^{5-7}$

The standard design approach for these structures is based on the idea that the membrane forms a series of small circular hoops which transmit the pressure load to the meridional tapes. It is unstressed in the meridional direction and hence it is likely to start wrinkling in this direction. The tapes are highly stressed and take up a "funicular" shape which is determined by the applied loads.

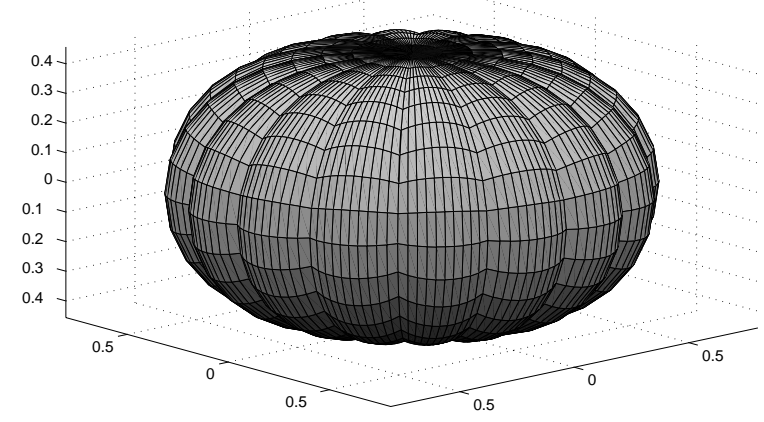

Figure 10: View of lobed spheroidal (nominal configuration)

The transverse lobe curvature, i.e. normal to the meridians, is assumed to be constant for equilibrium. The transverse radius of curvature need not be constant along the meridian, but it will be assumed to be so in the present study.
Profile of Meridional Tapes

This section is based on the work of Taylor ${ }^{3}$ with some minor modifications.

(a)

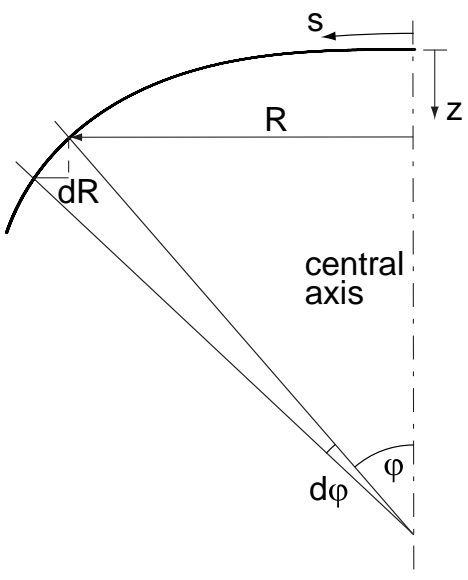

(b)

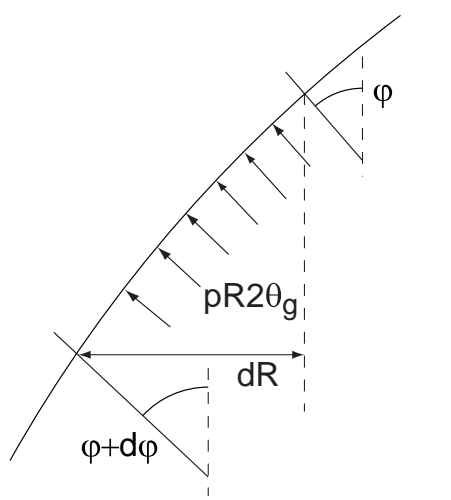

Figure 11: (a) Elevation of a meridian (b) detail of infinitesimal free body.

Vertical equilibrium of the infinitesimal element of meridian shown in Figure 11(a) yields

$$
\frac{d}{d R}(T R \sin \varphi)=p R
$$

where $\theta_{s}$ and $p$ are respectively the segment semiangle and the pressure and the other variables are as shown in Figure 11. Horizontal equilibrium gives

$$
\frac{d}{d R}(T R \cos \varphi)=-p R \tan \varphi
$$

It can then be shown that

$$
\frac{R^{2}}{R_{0}^{2}}=\sin \varphi
$$

where $R_{0}$ is the (maximum) horizontal radius of the meridian. 


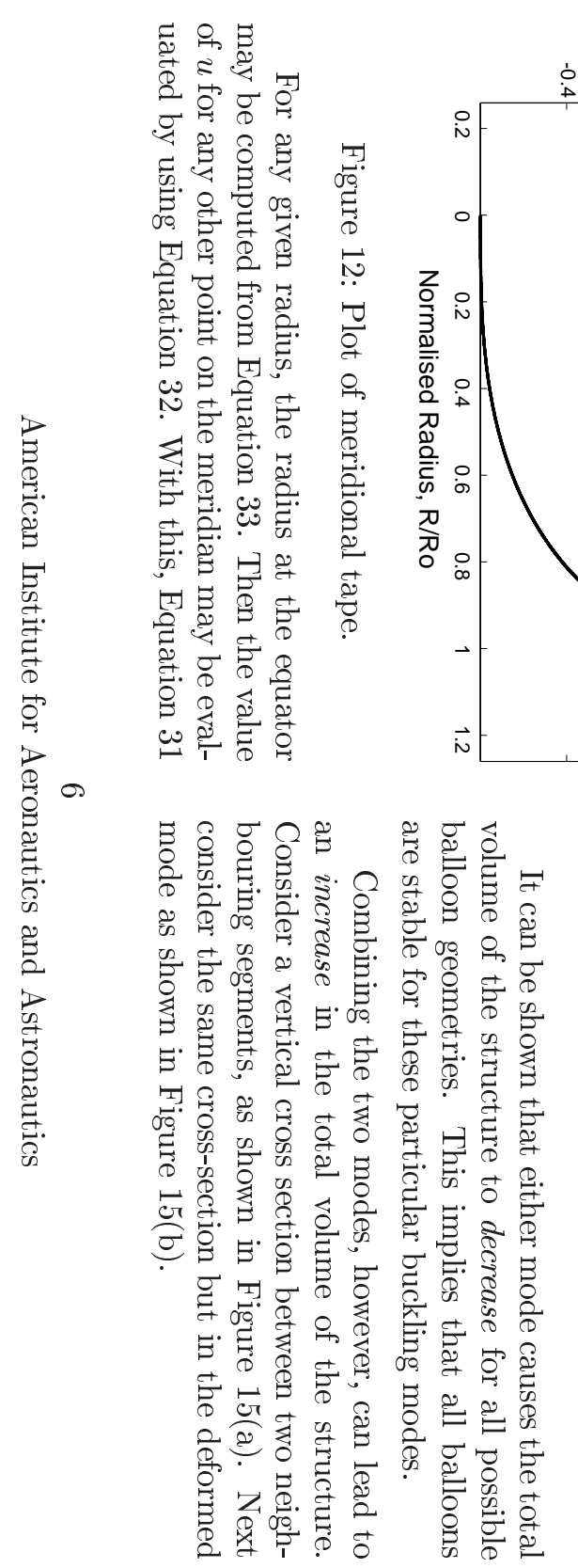

Normalised Height, $\mathrm{z}_{\mathrm{S}} / \mathrm{R}_{0}$
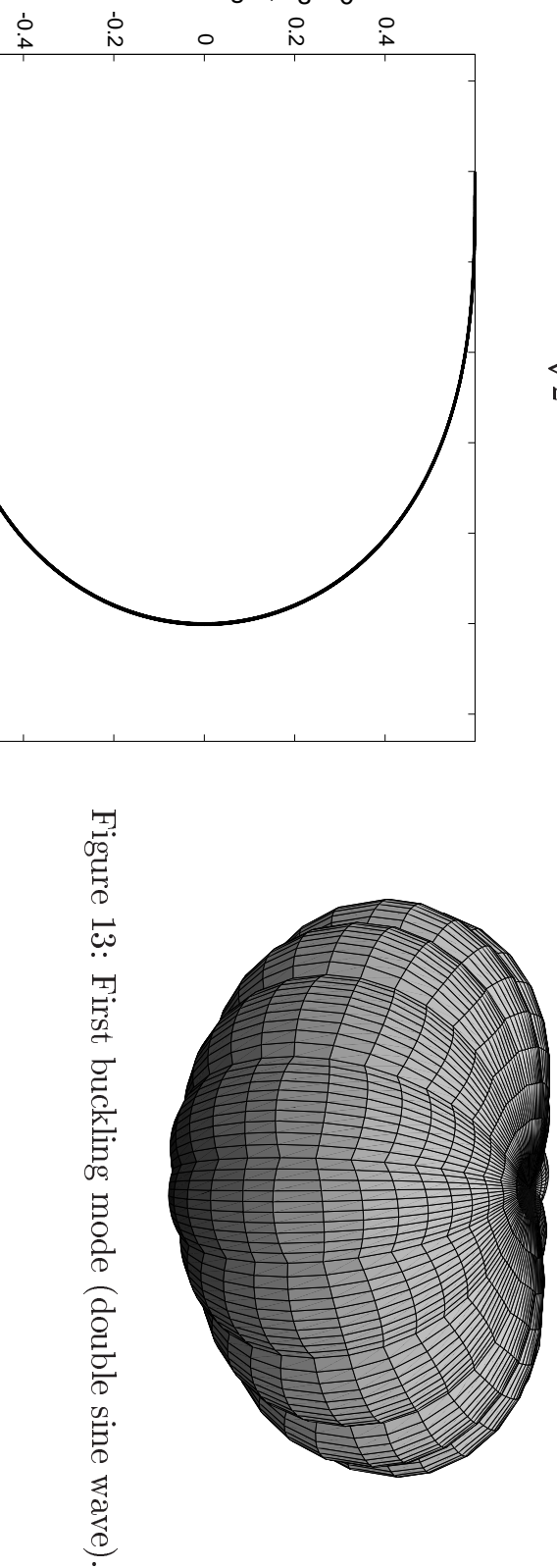

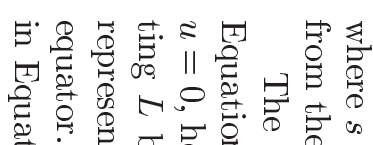

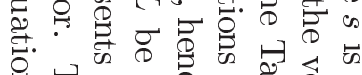

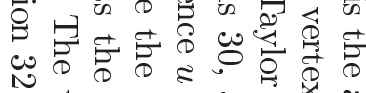

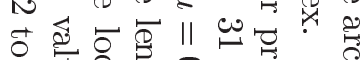

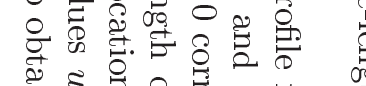

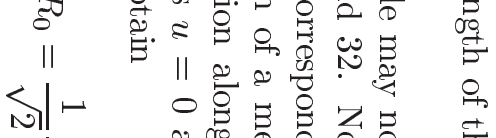

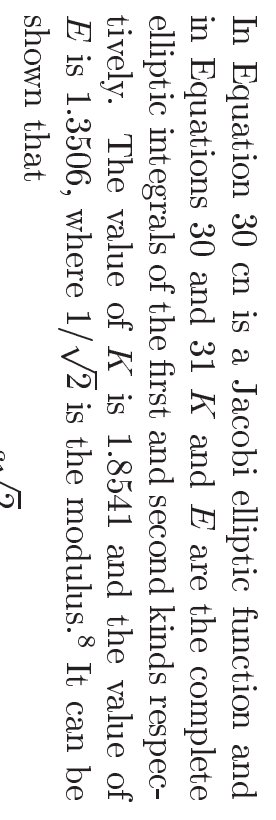

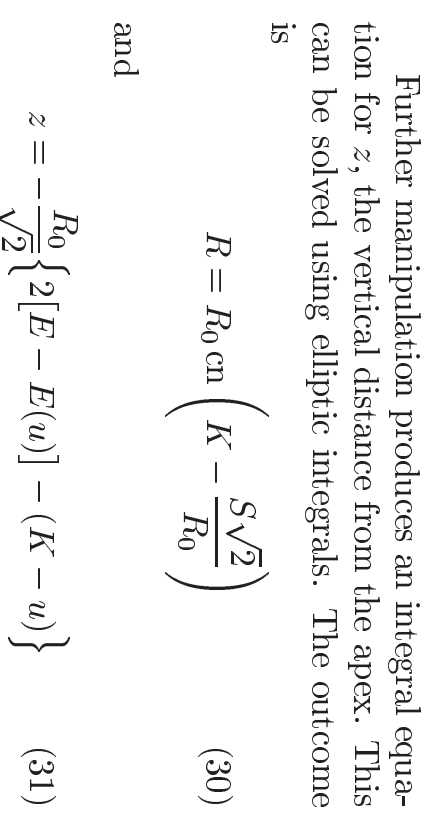

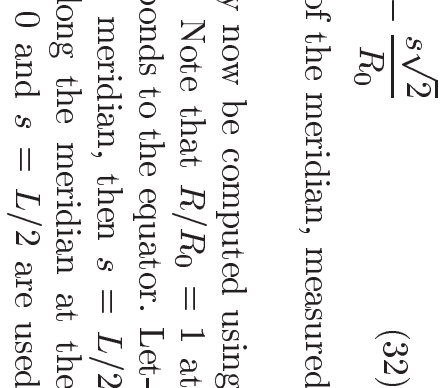

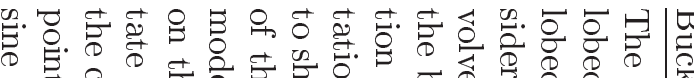

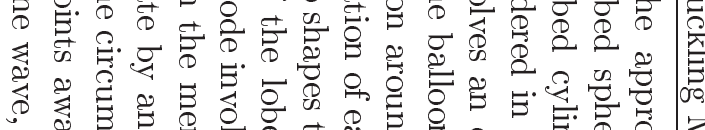

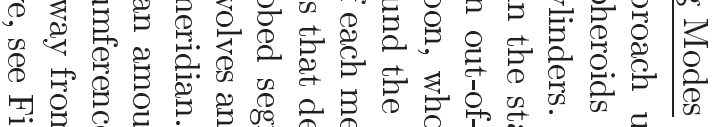

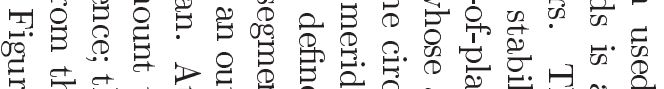

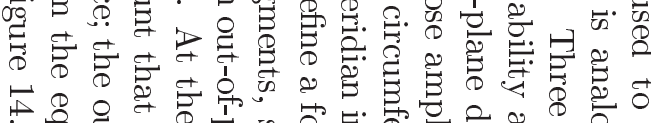

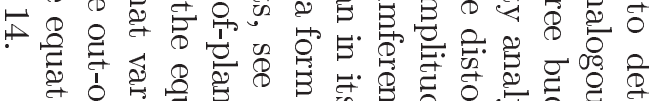

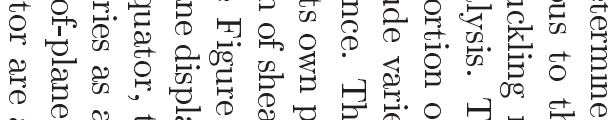

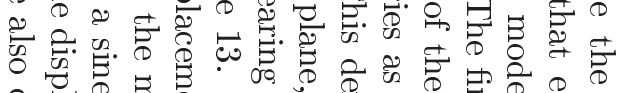

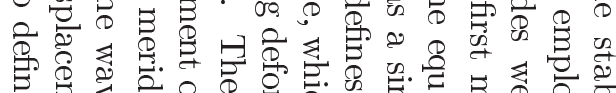

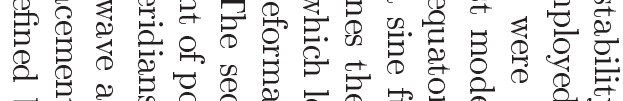

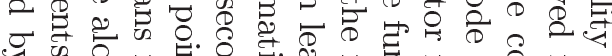

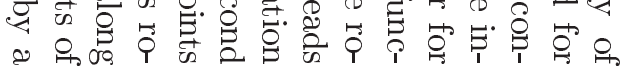

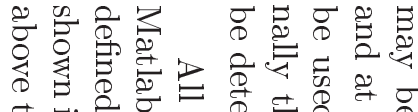

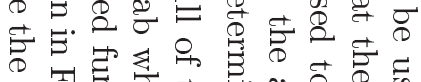

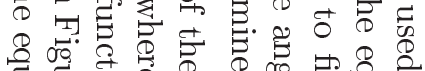

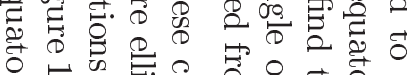

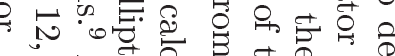

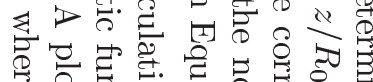

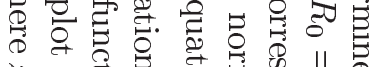

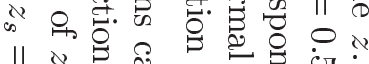

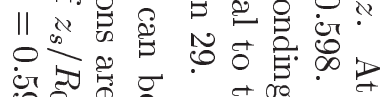

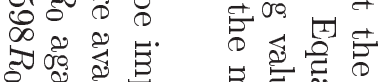

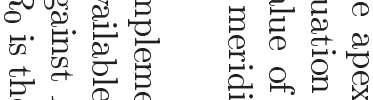

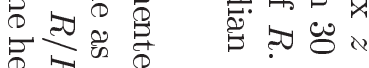

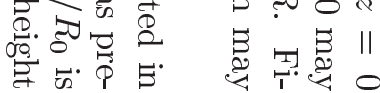




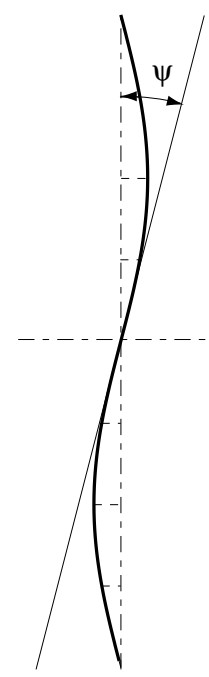

Figure 14: Edge-on view of a meridian in second buckling mode.

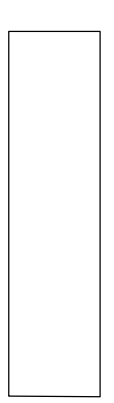

(a)

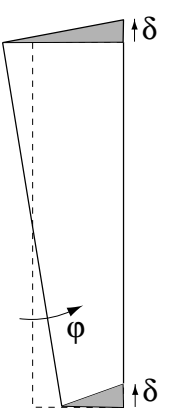

(b)
Figure 15: Vertical cross-section between meridians

The contribution from the first buckling mode can be represented by a vertical deformation $\delta$ of one side of the rectangle while the contribution from the second buckling mode can be represented by a rotation $\varphi$ of the other side. The area of the crosssection becomes greater in the deformed shape, as can be seen by comparing the areas that are lost with those gained (shaded in Figure 15). Because the volume of a whole balloon is, in effect, obtained by integrating over a series of elementary volumes with the cross-sections shown in Figure 15, we have thus shown that the volume enclosed in a balloon excluding the lobes - can be expected to increase for any combined mode. In fact, as we will see at the end of the paper, for small deformation amplitudes the volume of the segments decreases, due to the fact that in the first mode the meridians are being rotated. The volume enclosed by the lobes will decrease in the buckled configuration, but this is a less significant effect in some configurations.
A view of the meridians for the third buckling mode is shown in Figure 16 for a maximum deformation of $5^{\circ}$. Figure 17 presents the same structure, but with the lobes shown.

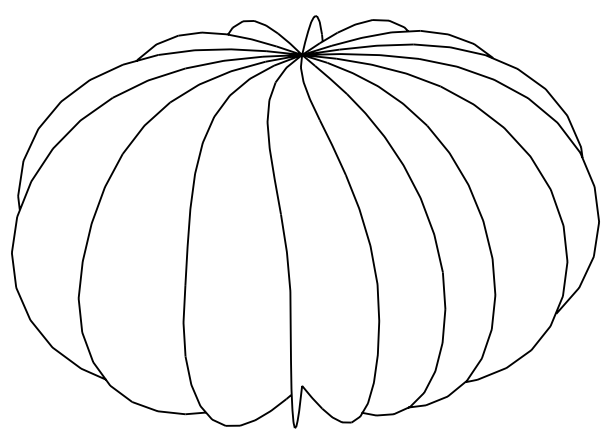

Figure 16: Meridians in third buckling mode.

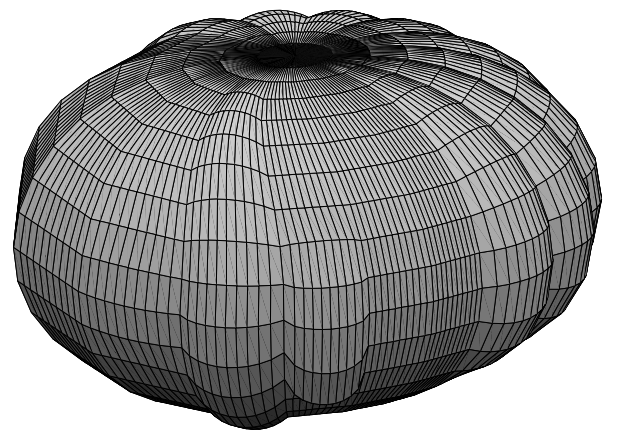

Figure 17: Third buckling mode.

\section{Volume Calculations}

The volumes of a number of different balloons with different numbers of segments were calculated using Matlab. ${ }^{9}$ The volume calculation algorithm is divided into two parts. The first part determines the volume of the segments assuming flat lobes. The input required to do this calculation is a set of $n$ points on each meridian, typically $n=21$ plus a single point on the central axis. These points define the vertices of $n-1$ pyramids, one of which is shown in Figure 18. Each pyramid is divided into two tetrahedra, as shown, whose volume can be calculated from

$$
\text { Volume }=\frac{1}{6}\left|\begin{array}{llll}
x_{1} & y_{1} & z_{1} & 1 \\
x_{2} & y_{2} & z_{2} & 1 \\
x_{3} & y_{3} & z_{3} & 1 \\
x_{4} & y_{4} & z_{4} & 1
\end{array}\right|
$$

where $\left(x_{i}, y_{i}, z_{i}\right)$ are the cartesian coordinates of point $i$. The volume of a whole segment is determined by summing the volumes of $n-1$ pyramids, and the volume of the whole balloon is the sum of the volumes of all of its segments. 


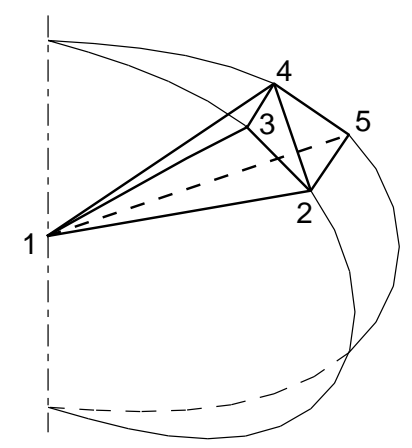

(a)

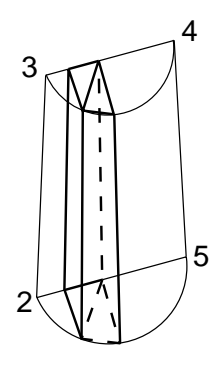

(b)
Figure 18: Volume discretisation scheme.

The second part of the volume calculation algorithm deals with the lobes. In analogy with the first part, $m$ points - typically $m=11$ - are located on the circular arc that defines a section of the lobe passing through two corresponding points on neighbouring meridians. In addition, points are defined on corresponding chords, Figure 18(b). The volume enclosed by this part of the lobe is obtained, again, by summing the volumes of several tetrahedra. The volume of a whole lobe is obtained by summing $m-1$ contributions.

Calculations were performed for balloons with sixteen, thirty-two and sixty-four meridians with $L=2$, for three different lobe radii of curvature $R_{l}$. Table 1 presents the results for the undeformed case. Table 2 presents the results for the third type of buckling mode where the deformation varies as a single sine wave around the circumference. Tables 3 and 4 present the results for deformation modes based on double and quadruple sine waves respectively. The results are presented for four different amplitudes of the buckling mode. Note that the upper-right part of each table is empty, because the smaller values of $R_{l} / L$ are not admissible for balloons with a small number of meridians.

\begin{tabular}{|c|c|c|c|}
\hline Number of & \multicolumn{3}{|c|}{$R_{l} / L$} \\
\cline { 2 - 4 } Meridians & 0.4 & 0.2 & 0.1 \\
\hline 16 & 1.3359 & - & - \\
\hline 32 & 1.2330 & 1.2784 & - \\
\hline 64 & 1.2125 & 1.2214 & 1.2439 \\
\hline
\end{tabular}

Table 1: Volume of undeformed structure.

\begin{tabular}{|c|c|c|c|c|}
\hline Number of & Ampl. & \multicolumn{3}{|c|}{$R_{l} / L$} \\
\cline { 2 - 5 } Meridians & $\left({ }^{\circ}\right)$ & 0.4 & 0.2 & 0.1 \\
\hline \multirow{4}{*}{16} & 0.5 & 1.3330 & - & - \\
\cline { 2 - 5 } & 1.0 & 1.3259 & - & - \\
\cline { 2 - 5 } & 2.0 & 1.3105 & - & - \\
\cline { 2 - 5 } & 5.0 & 1.2644 & - & - \\
\hline 32 & 0.5 & 1.2282 & 1.2768 & - \\
\cline { 2 - 5 } & 1.0 & 1.2204 & 1.2732 & - \\
\cline { 2 - 5 } & 2.0 & 1.2158 & 1.2648 & - \\
\cline { 2 - 5 } & 5.0 & 1.2088 & 1.2417 & - \\
\hline \multirow{6}{*}{6} & 0.5 & 1.2085 & 1.2189 & 1.2431 \\
\cline { 2 - 5 } & 1.0 & 1.2078 & 1.2150 & 1.2412 \\
\cline { 2 - 5 } & 2.0 & 1.2068 & 1.2126 & 1.2368 \\
\cline { 2 - 5 } & 5.0 & 1.2026 & 1.2075 & 1.2237 \\
\hline
\end{tabular}

Table 2: Volume of structure deformed in a single sine wave.

\begin{tabular}{|c|c|c|c|c|}
\hline Number of & Ampl. & \multicolumn{3}{|c|}{$R_{l} / L$} \\
\cline { 2 - 5 } Meridians & $\left({ }^{\circ}\right)$ & 0.4 & 0.2 & 0.1 \\
\hline \multirow{4}{*}{16} & 0.5 & 1.3107 & - & - \\
\cline { 2 - 5 } & 1.0 & 1.2727 & - & - \\
\cline { 2 - 5 } & 2.0 & 1.2492 & - & - \\
\cline { 2 - 5 } & 5.0 & 1.3508 & - & - \\
\hline 32 & 0.5 & 1.2166 & 1.2660 & - \\
\cline { 2 - 5 } & 1.0 & 1.2116 & 1.2488 & - \\
\cline { 2 - 5 } & 2.0 & 1.2068 & 1.2358 & - \\
\cline { 2 - 5 } & 5.0 & 1.3623 & 1.3935 & - \\
\hline 64 & 0.5 & 1.2072 & 1.2132 & 1.2374 \\
\cline { 2 - 5 } & 1.0 & 1.2065 & 1.2114 & 1.2294 \\
\cline { 2 - 5 } & 2.0 & 1.2043 & 1.2088 & 1.2226 \\
\cline { 2 - 5 } & 5.0 & 1.3759 & 1.3801 & 1.3884 \\
\hline
\end{tabular}

Table 3: Volume of structure deformed in a double sine wave.

\begin{tabular}{|c|c|c|c|c|}
\hline Number of & Ampl. & \multicolumn{3}{|c|}{$R_{l} / L$} \\
\cline { 2 - 5 } Meridians & $\left({ }^{\circ}\right)$ & 0.4 & 0.2 & 0.1 \\
\hline \multirow{4}{*}{16} & 0.5 & 1.2382 & - & - \\
\cline { 2 - 5 } & 1.0 & 1.2055 & - & - \\
\cline { 2 - 5 } & 2.0 & 1.5539 & - & - \\
\cline { 2 - 5 } & 5.0 & 1.9897 & - & - \\
\hline 32 & 0.5 & 1.2076 & 1.2359 & - \\
\cline { 2 - 5 } & 1.0 & 1.2768 & 1.2977 & - \\
\cline { 2 - 5 } & 2.0 & 1.6137 & 1.6335 & - \\
\cline { 2 - 5 } & 5.0 & 2.5510 & 2.5776 & - \\
\hline \multirow{6}{*}{6} & 0.5 & 1.2058 & 1.2094 & 1.2239 \\
\cline { 2 - 5 } & 1.0 & 1.2809 & 1.2847 & 1.2964 \\
\cline { 2 - 5 } & 2.0 & 1.7119 & 1.7150 & 1.7252 \\
\cline { 2 - 5 } & 5.0 & 2.9466 & 2.9490 & 2.9570 \\
\hline
\end{tabular}

Table 4: Volume of structure deformed in a quadruple sine wave.

A comparison of the volumes of the deformed balloon, Tables $2-4$, with the volumes of the corre- 
sponding balloons in the nominal configuration, Table 1 , shows that the single sine wave mode leads to smaller volumes. The modes with higher wave numbers produce a volume increase for sufficiently large amplitudes.

\section{$\underline{\text { Discussion }}$}

Prior to the study presented in this paper there was little evidence of instability in membrane structures. The Endeavour balloon provided physical proof that instability could be a problem and this was the main inspiration for this work.

Both of the structures investigated in this paper show unstable behaviour. The analysis of the lobed cylinder demonstrates that a simple membrane structure becomes unstable for certain parameters. The system stability may be determined by examining just three parameters, namely the number of lobes $n$, the overall aspect ratio $a$ and the half-angle $\theta$ embraced by the lobes in cross-section.

The lobed spheroid is more complex and its unstable deformation modes are more subtle. A deformation mode was proposed which was found to produce unstable behaviour. An interesting feature of this mode is that the enclosed volume initially decreases with increasing deformation. This feature was investigated further by calculating separately the lobe and segment volumes. Table 5 shows the results for balloons with $R_{l} / L=0.4$ and $L=2$, that are deformed into a double sine wave.

The volume of the segments initially decreases until there is sufficient deformation to result in an increase in the segment volume. The volume of the lobes decreases monotonically. The total volume initially decreases before increasing until such time that it is greater than the initial volume. At this point it may be said that the structure is more stable in the deformed mode.

The relative stability of the deformed shapes may be seen more clearly if the volumes are normalised relative to the corresponding nominal volume. Table 6 presents these values for a double sine wave mode.

It is clear that the change in total volume and hence the stability of the balloon is closely linked to the number of meridians. The greater the number of meridians the smaller the amount of deformation required to cause the total volume to increase. This is shown in Figure 19 which is a plot of the normalised volumes for $R_{l} / L=0.4$.

\begin{tabular}{|c|c|c|c|c|}
\hline \multirow{2}{*}{$\begin{array}{l}\text { Number of } \\
\text { Meridians }\end{array}$} & \multirow{2}{*}{$\begin{array}{c}\text { Ampl. } \\
\left({ }^{\circ}\right)\end{array}$} & \multicolumn{3}{|c|}{ Volume } \\
\hline & & Segments & Lobes & Total \\
\hline \multirow[t]{5}{*}{16} & 0 & 1.1753 & 0.1606 & 1.3359 \\
\hline & 0.5 & 1.1746 & 0.1361 & 1.3107 \\
\hline & 1.0 & 1.1724 & 0.1003 & 1.2727 \\
\hline & 2.0 & 1.1634 & 0.0858 & 1.2492 \\
\hline & 5.0 & 1.2836 & 0.0672 & 1.3508 \\
\hline \multirow[t]{5}{*}{32} & 0 & 1.1983 & 0.0346 & 1.2330 \\
\hline & 0.5 & 1.1981 & 0.0185 & 1.2166 \\
\hline & 1.0 & 1.1973 & 0.0143 & 1.2116 \\
\hline & 2.0 & 1.1940 & 0.0127 & 1.2068 \\
\hline & 5.0 & 1.3524 & 0.0099 & 1.3623 \\
\hline \multirow[t]{5}{*}{64} & 0 & 1.2041 & 0.0084 & 1.2125 \\
\hline & 0.5 & 1.2040 & 0.0032 & 1.2072 \\
\hline & 1.0 & 1.2036 & 0.0029 & 1.2065 \\
\hline & 2.0 & 1.2018 & 0.0024 & 1.2043 \\
\hline & 5.0 & 1.3742 & 0.0017 & 1.3759 \\
\hline
\end{tabular}

Table 5: Volume of structure deformed in a double sine wave, $R_{l} / L=0.4$.

\begin{tabular}{|c|c|c|c|c|}
\hline Number of & Ampl. & \multicolumn{3}{|c|}{$R_{l} / L$} \\
\cline { 2 - 5 } Meridians & $\left({ }^{\circ}\right)$ & 0.4 & 0.2 & 0.1 \\
\hline \multirow{5}{*}{16} & 0.5 & 0.9811 & - & - \\
\cline { 2 - 5 } & 1.0 & 0.9527 & - & - \\
\cline { 2 - 5 } & 2.0 & 0.9351 & - & - \\
\cline { 2 - 5 } & 5.0 & 1.0112 & - & - \\
\hline \multirow{5}{*}{32} & 0.5 & 0.9867 & 0.9903 & - \\
\cline { 2 - 5 } & 1.0 & 0.9826 & 0.9768 & - \\
\cline { 2 - 5 } & 2.0 & 0.9788 & 0.9667 & - \\
\cline { 2 - 5 } & 5.0 & 1.1049 & 1.0900 & - \\
\hline \multirow{5}{*}{64} & 0.5 & 0.9956 & 0.9933 & 0.9948 \\
\cline { 2 - 5 } & 1.0 & 0.9951 & 0.9918 & 0.9883 \\
\cline { 2 - 5 } & 2.0 & 0.9932 & 0.9897 & 0.9829 \\
\cline { 2 - 5 } & 5.0 & 1.1348 & 1.1299 & 1.1162 \\
\hline
\end{tabular}

Table 6: Normalised volume of structure deformed in a double sine wave.

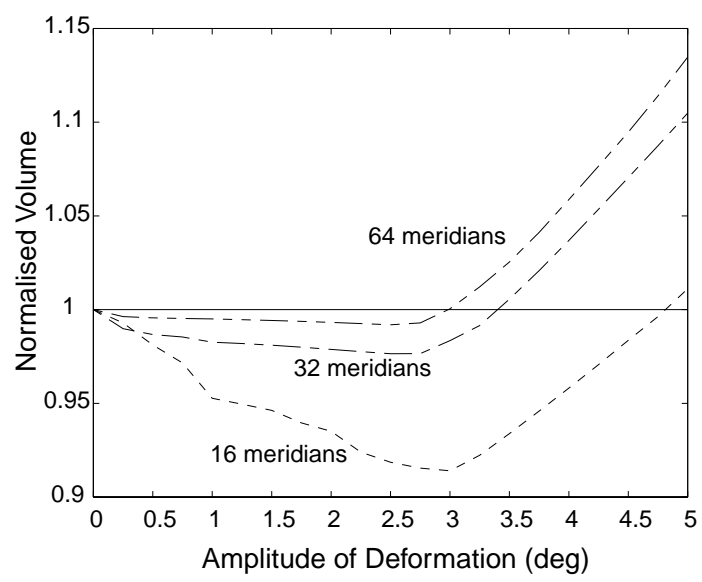

Figure 19: Variation of normalised volume with deformation amplitude $\left(R_{l} / L=0.4, L=2\right)$. 
The value of $R_{l}$, the transverse radius of curvature of the lobes, also has an effect on the total volume but this is less noticeable. Examination of Table 5 shows that the volume of the segments is much greater than the volume of the lobes and hence the lobes do not have as significant an effect as the number of meridians.

\section{$\underline{\text { Conclusion }}$}

It has been shown that both a simple lobed column and a lobed spheroid may become unstable. The stability of the lobed column depends entirely on its geometry. The stability of the spheroid depends both on the geometry of the structure and on a small but finite perturbation. The size of the perturbation required to cause the structure to become unstable depends closely on the deformation mode and on the number of meridians in the structure. The larger the number of meridians the smaller the perturbation required to cause instability. The effect of the lobe geometry is not as significant as for the column.

\section{Acknowledgment}

The authors would like to thank Prof C.R. Calladine for many helpful comments on the work.

\section{Bibliography}

1. Spencer, D.A. et al. Mars Pathfinder entry, descent, and landing reconstruction, J. Spacecraft Rockets, Vol. 36, 1999.
2. Calladine, C.R. Stability of the Endeavour balloon, in Buckling of Structures (edited by I. Elishakoff et al.), Elsevier Science Publishers, 1988.

3. Taylor, G.I. On the Shapes of Parachutes (Paper written for the Advisory Committee on Aeronautics), in The Scientific Papers of G.I. Taylor (1963) (edited by G.K. Batchelor), Vol. 3, Cambridge University Press, Cambridge.

4. Mikulas, M. M. and Bohon, H.L. Development status of attached inflatable decelerators, Journal of Spacecraft and Rockets, Vol 6, 1969.

5. Yajima, N., Izutsu, N., Honda, H., Kurokawa, H. and Matsushima, K. A new design concept of natural shape balloon for high pressure durability, AIAA International Balloon Technology Conference, June 28-July 1 1999, Norfolk, VA.

6. Schur, W. W. Analysis of load tape constrained pneumatic envelopes, 40th AIAA/ASME/ASCE/AHS/ASC Structures, Structural Dynamics and Materials Conference, April 1999, St Louis, MO.

7. Baginski, F. and Brakke, K.A. Modeling ascent configurations of strained high-altitude balloons, AIAA Journal, Vol. 36, 1998.

8. Abramowitz, M. and Stegun, I.A. Handbook of Mathematical Functions, Dover, New York, 1965 .

9. Mathworks Inc. Using Matlab, Mathworks Inc, Natick USA, 1996. 